\title{
Correlation between GEM Premier 3000 and Vitros5.1+5600, SYSMEX XN-9000 in Detecting Electrolytes and Red Blood Cell Volume
}

\author{
Weihua Xue, Shunling Li
}

Department of Critical Care Medicine, The First Affiliated Hospital of Sun Yat-Sen University, Guangzhou, China

Correspondence to: Shunling Li, lishling@mail.sysu.edu.cn

Keywords: Blood Gas Analyzer, Serum Potassium, Serum Sodium, Blood Glucose, Hemoglobin, Red Blood Cell

Volume, Central Laboratory

Received: December 10, $2021 \quad$ Accepted: January 25, $2022 \quad$ Published: January 28, 2022

Copyright $\odot 2022$ by author(s) and Scientific Research Publishing Inc.

This work is licensed under the Creative Commons Attribution International License (CC BY 4.0).

http://creativecommons.org/licenses/by/4.0/

\section{(c) (1) Open Access}

\section{ABSTRACT}

Objective: To explore the consistency and relevance of the results of the bedside rapid blood gas analyzer GEM premier 3000, the Vitros5.1+5600 biochemical immunoassay analyzer and the SYSMEX XN-9000 automatic blood cell analyzer in the central laboratory detecting serum potassium $\left(\mathrm{K}^{+}\right)$, serumsodium $\left(\mathrm{Na}^{+}\right)$, blood glucose $(\mathrm{Glu})$, hemoglobin $(\mathrm{Hb})$ and red blood cell volume ( $\mathrm{Hct})$. And to provide a reference for the accurate interpretation of the bedside blood gas analysis report. Method: Usually, ICU patients will be taken arterial blood gas, biochemical and blood samples through the arterial indwelling needle; at the same time patients' potassium, serum sodium, blood glucose, hemoglobin and red blood cell volume will be detected. This study implemented paired t-test and correlation regression analysis on each group of data, and used the analysis quality requirements (allowable total error) of CLIA'88 proficiency testing program as the criteria for clinical acceptance. Results: The paired t-test showed that the serum potassium, serum sodium and blood glucose detected by GEM premier 3000 and Vitros5.1+5600 were significantly different; and the hemoglobin and red blood cell volume detected by GEM premier 3000 and SYSMEX XN-9000 were significantly different $(P<0.05)$. The Pearson correlation coefficients $(r)$ of hemoglobin, red blood cell volume and red blood cell volume were $0.860,0.886,0.924,0.841$ and 0.856 , respectively, and the above test items all had good correlations $(P<0.05)$. The average $(\mathrm{SE})$ of the paired differences of $\mathrm{K}^{+}, \mathrm{Na}^{+}, \mathrm{Glu}, \mathrm{Hb}$ and $\mathrm{Hct}$ detected by the two sets of instruments is less than the allowable error of CLIA'88, and the SE of blood $\mathrm{Na}^{+}$and $\mathrm{Hb}$ is less than half of the allowable error of CLIA'88. Conclusion: The test results of GEM premier 3000, the central laboratory Vitros5.1+5600 and SYSMEX XN-9000 have good correlation, but the con- 


\section{sistency is not good. The test results of GEM premier 3000 cannot replace the central labor-}

atory.

\section{INTRODUCTION}

The bedside rapid blood gas analyzer can get bleeding gas results in one minute, which makes it possible to realize rapid diagnosis and treatment for critically ill patients in the ICU, and brings convenience to patients and clinics. However, as the types of blood gas analyzers and test items are gradually increasing, problems emerge. Due to the differences in test methods and instruments, the results of testing the same items at the same time are different; besides there are also differences between the rapid blood gas analyzer and the central laboratory [1-3]. How do clinical medical staff and patients view this difference, whether the differences have regularity and what are the influencing factors? In current studies, there are many comparisons of arterial blood and venous blood. However, with the widespread use of intra-arterial indwelling needles in the ICU for dynamic monitoring of patients' blood pressure [4], only arterial specimens are collected. This study intends to compare the differences and correlations of the results that 3 instruments (the clinical GEM premier 3000 blood gas analyzer, the central laboratory Vitros5.1+5600 biochemical immunoassay analyzer and the SYSMEX XN-9000 automatic blood cell analyzer) produce at the same time from detecting the same items in arterial specimens (potassium, serum sodium, blood glucose, hemoglobin, hemoglobin and red blood cell volume) and provide a reference for the accurate interpretation of the report of the bedside blood gas analyzer GEM premier 3000.

\section{MATERIALS AND METHODS}

\subsection{Clinical Information}

Doctors will prescribe medical orders for biochemical, blood routine, and arterial blood gas tests in the morning based on the conditions of the ICU patients. All samples of this study were executed according to the doctor's orders and did not cause any additional damage to the patients. This study has been exempted from signing the informed consent form by the Scientific Research Management Office of the hospital. The collection method is to collect arterial blood gas, biochemical and blood routine samples at one time through the arterial catheter. The data collection uses questionnaires and trains a researcher to collect data. A total of 322 samples are collected from the ward in December 2020. We performed basic logic (the difference between the results of the same item was greater than 50\%) and omission analysis on the data, and excluded 5 invalid questionnaires, resulting in a total of 317 valid questionnaires. Among them, 235 cases were male (74.1\%), 82 cases were female (25.9\%), and the age was $50.0 \pm 15.6$ (18 - 93).

\subsection{Instrument and Detection Principle}

GEM premier 3000 blood gas analyzer detects arterial blood gas analysis. Vitros $5.1+5600$ biochemical immunoassay analyzer detects biochemical indicators. SYSMEX XN-9000 automatic blood cell analyzer detects blood routine indicators. The accessories of these instruments are all supporting products. The instruments have met the requirements of quality control management and can be used for clinical specimen testing. GEM premier 3000 uses the direct electrode method to detect serum potassium and serum sodium, immediately after the specimen is collected; Vitros5.1+5600 uses the direct electrode method to detect serum potassium and serum sodium, which is detected approximately 1 hour after the specimen is collected. GEM premier 3000 detects blood glucose by directly measuring the change in current when glucose oxidase reacts with blood glucose to produce hydrogen peroxide; Vitros5.1+5600 detects blood glucose using a multi-layer coating dry chemical method. GEM premier 3000 detects red blood cell volume using the conductive cell method to calculate the value of serum sodium, and hemoglobin is calculated using the built-in formula (red blood cell volume*coefficient). SYSMEX XN-9000 uses centrifugal method to detect red blood cell volume and hemoglobin. 


\subsection{Statistical Analysis}

SPSS23.0 software was used in data analysis which included paired $t$ test and correlation coefficient ( $r$ ) analysis on the measurement results of the two detection instruments. $\mathrm{P}<0.05$ indicated that the difference was statistically significant. Referring to the contents of the CLSI EP9-A3 document, we compared the systematic error (SE) of the measured data of the two instruments, $\mathrm{SE}=|\mathrm{YX}|$. We took the analysis quality requirements (allowable total error) of the US CLIA'88 proficiency testing program as the standard of inspection capability.

\section{RESULTS}

1) Analysis of Differences

A paired t test was conducted on two groups of data, and the results showed that the differences of the two groups of serum potassium, serum sodium, blood glucose, hemoglobin and red blood cell volume were statistically significant $\mathrm{P}<0.05$, see Table 1 . It shows that the results of GEM premier 3000 in detecting serum potassium, serum sodium, blood glucose, hemoglobin and red blood cell volume are different from those of the central laboratory Vitros5.1+5600 and SYSMEX XN-9000, and cannot replace the results of the central laboratory clinically.

2) Correlation Analysis

According to the performance reports of Vitros5.1+5600 biochemical immunoassay analyzer and SYSMEX XN-9000 automatic blood cell analyzer, the two systems have the characteristics of high precision, high accuracy and good repeatability $[5,6]$, so they are used as Reference system (X); GEM premier 3000 is used as the system under test $(\mathrm{Y})$. The two sets of data are performed regression correlation analysis. The regression equation and correlation coefficients are showed in Table 2, and the scatter diagram in Figure 1. The results showed that the two groups of serum potassium, serum sodium, blood sugar, hemoglobin and red blood cell volume are correlated $\mathrm{P}<0.05$.

3) Compared with the analysis quality requirements (allowed total error) of the American CLIA'88 proficiency testing program [7]: Referring to CLSI EP9-A3 document [8], we compared the average (SE, system error) of the two testing systems, and performed a paired t test on the two sets of data to obtain whether the SE of the paired serum potassium, serum sodium, blood glucose, hemoglobin and red blood cell volumes are within the allowable range of CLIA'88, see Table 3. The results showed that the systematic error SE of the two groups of serum potassium, serum sodium, blood glucose and hemoglobin were within the allowable range of CLIA'88, while the red blood cell volume was not.

Table 1. Paired t test between GEM premier 3000 blood gas analyzer and laboratory equipment $(\mathrm{n}=$ 317).

\begin{tabular}{ccccc}
\hline project & GEM premier 3000 & Central laboratory & $\mathrm{t}$ & $\mathrm{p}$ \\
\hline serum potassium (mmol/L) & $4.02 \pm 0.45$ & $4.32 \pm 0.46$ & -22.642 & $0.000^{*}$ \\
Blood sodium (mmol/L) & $138.27 \pm 5.23$ & $140.24 \pm 5.48$ & -12.934 & $0.000^{*}$ \\
Blood sugar (mmol/L) & $8.98 \pm 3.03$ & $8.67 \pm 3.19$ & 4.379 & $0.000^{*}$ \\
Hemoglobin (g/L) & $91.01 \pm 16.82$ & $93.31 \pm 16.83$ & -4.299 & $0.000^{*}$ \\
red blood cell volume (\%) & $29.25 \pm 5.43$ & $28.28 \pm 4.92$ & 6.140 & $0.000^{\star}$ \\
\hline
\end{tabular}

Note: ${ }^{*}$ means $\mathrm{P}<0.05$, and the correlation is statistically significant. 
Table 2. Regression equation and Pearson correlation coefficients $(n=317)$.

\begin{tabular}{cccc}
\hline project & Regression equation & $\begin{array}{c}\text { Pearson correlation } \\
\text { coefficient } \mathrm{r}\end{array}$ & $\mathrm{P}$ \\
\hline serum potassium $(\mathrm{mmol} / \mathrm{L})$ & $\mathrm{Y}=0.884 \mathrm{X}+0.772$ & 0.860 & $0.000^{*}$ \\
Blood sodium $(\mathrm{mmol} / \mathrm{L})$ & $\mathrm{Y}=0.930 \mathrm{X}+11.62$ & 0.886 & $0.000^{*}$ \\
blood sugar $(\mathrm{mmol} / \mathrm{L})$ & $\mathrm{Y}=0.972 \mathrm{X}-0.054$ & 0.924 & $0.000^{*}$ \\
hemoglobin $(\mathrm{g} / \mathrm{L})$ & $\mathrm{Y}=0.840 \mathrm{X}+12.638$ & 0.841 & $0.000^{*}$ \\
red blood cell volume $(\%)$ & $\mathrm{Y}=0.943 \mathrm{X}+2.579$ & 0.856 & $0.000^{*}$ \\
\hline
\end{tabular}

Note: ${ }^{\star}$ means $\mathrm{P}<0.05$, and the correlation is statistically significant.

Table 3. Comparison of the difference between the two sets of paired data and the allowed range of CLIA'88 ( $\mathrm{n}=317)$.

\begin{tabular}{cccc}
\hline project & SE & CLIA'88 allowable error & Is it acceptable \\
\hline serum potassium $(\mathrm{mmol} / \mathrm{L})$ & 0.30 & $\pm 0.5 \mathrm{mmol} / \mathrm{L}$ & yes \\
serum sodium $(\mathrm{mmol} / \mathrm{L})$ & 1.87 & $\pm 4 \mathrm{mmol} / \mathrm{L}$ & yes \\
blood sugar $(\mathrm{mmol} / \mathrm{L})$ & 0.30 & $\pm 0.33 \mathrm{mmol} / \mathrm{L}(6 \mathrm{mg} / \mathrm{dL})$ or $\pm 10 \%$ & yes \\
hemoglobin $(\mathrm{g} / \mathrm{L})$ & 2.30 & $( \pm 7 \%) 6.53$ & yes \\
red blood cell volume $(\%)$ & 0.97 & $( \pm 6 \%) 1.70$ & yes \\
\hline
\end{tabular}

\section{DISCUSSION}

With the continuous update of medical equipment, more test items can be tested on different types of instruments, compared with the central laboratory examination, the rapid blood gas analyzer has an absolute advantage in terms of speed, and it is routinely configured in the ICU and emergency department. This study shows that the differences in serum potassium, serum sodium, blood glucose, hemoglobin, and red blood cell volume detected by GEM premier 3000, Vitros5.1+5600 and SYSMEX XN-9000 are statistically significant, which is consistent with some domestic and foreign research results [1-3]. For abnormal results of blood gas analysis, if necessary, the central laboratory must be rechecked. Through correlation analysis, the correlation of the above test items is statistically significant. At the same time, it meets the analysis quality requirements of CLIA'88 proficiency testing program (total error allowed), indicating that in emergency situations, bedside blood gas analysis is useful for disease judgment. The research method of this study is close to the actual clinical process, generally in the morning, specimens are collected and sent for inspection uniformly, which delays the test time of specimens by about 1 hour. Therefore, this study has practical significance for interpreting the test results of the blood gas analyzer and the central laboratory.

Compared with the research of Yu Jianhong et al. [9-11], the average values of serum potassium and sodium measured by GEM premier 3000 are lower than the central laboratory, with consistent results. Generally, serum potassium is low by about $0.1-0.3 \mathrm{mmol} / \mathrm{L}$ and serum sodium is low by $1-4 \mathrm{mmol} / \mathrm{L}$, because the heparin solution in the arterial blood sampling needle combines with $\mathrm{K}^{+}$and $\mathrm{Na}^{+}$; in the process of oscillating transportation and centrifugal separation of the specimens, the non-obvious hemolysis leads to ion leakage. The correlation coefficients of serum potassium and serum sodium in this study 

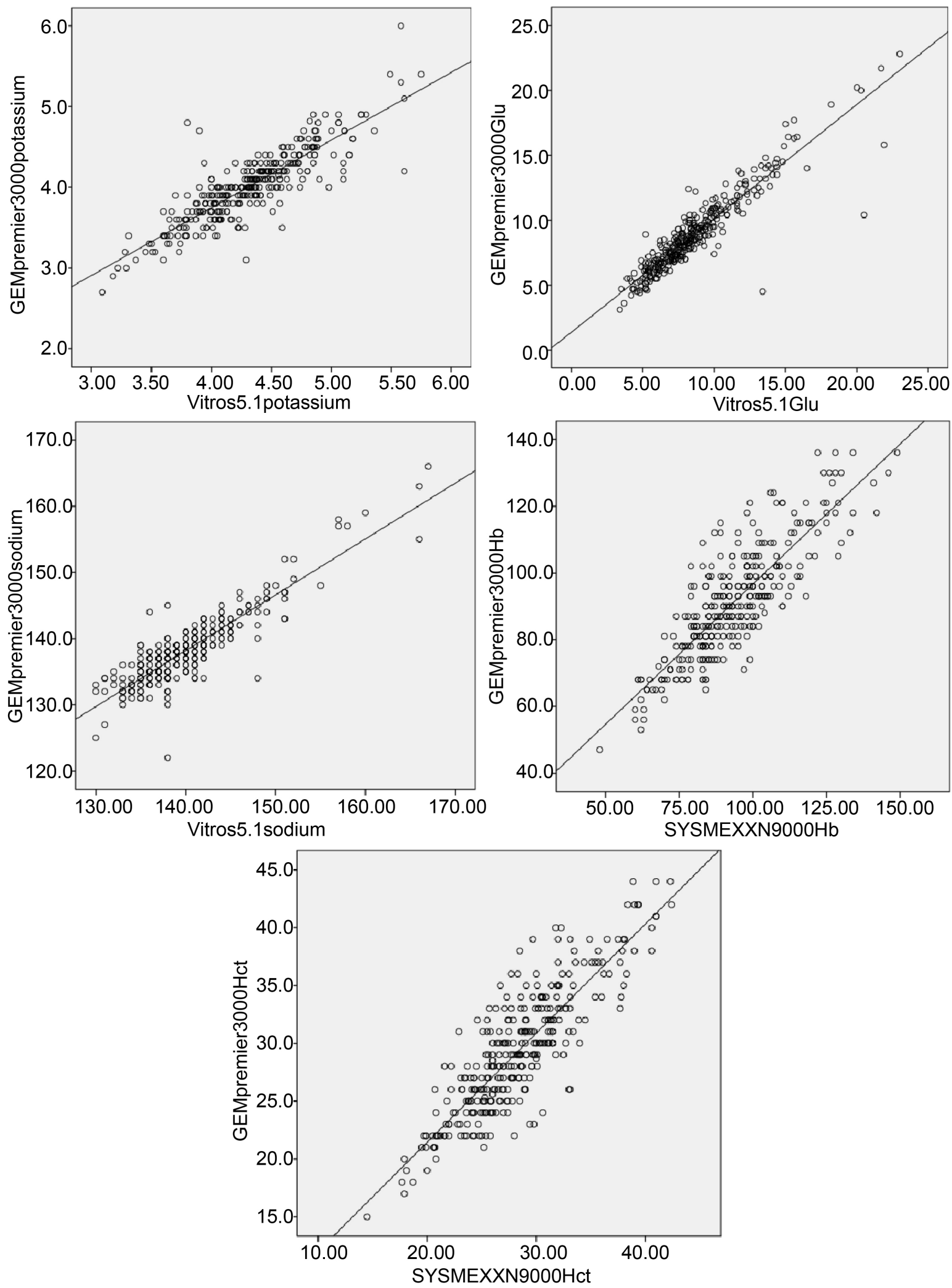

Figure 1. Scatter diagram of the test results of the two instruments $(n=317)$. Note: The detection result of GEM premier 3000 is in the $Y$ axis, and the detection result of Vitros5.1+5600 and SYSMEX $\mathrm{XN}-9000$ is in the $\mathrm{X}$ axis. 
are 0.88 and 0.93 , which are similar to the results of Yu Jianhong [9], indicating that the correlation between serum sodium is better in the same artery specimen.

The average blood glucose measured by GEM premier 3000 in this study is about $0.3 \mathrm{mmol} / \mathrm{L}$ higher than the central laboratory. It is inconsistent with the results of Xiong Wufang et al. $[10,12]$ that are as low as about $0.05 \mathrm{mmol} / \mathrm{L}$. The possible reason is that the biochemical test specimens in this study were delayed for about 1 hour, but the blood gas analysis test was performed immediately. The above studies are biochemical and blood gas tests at the same time, indicating that over time, red blood cells will ferment blood glucose, resulting in a decrease in blood glucose. Therefore, the biochemical test collected in the morning may cause the blood glucose to be lower than the actual value. The correlation coefficient of blood glucose in this study is 0.97 , which is consistent with the results of other studies, indicating that the correlation of blood glucose is good.

Compared with the research of Zhang Lei et al. $[2,3,10,13]$, the average red blood cell volume detected by GEM premier 3000 is higher than the central laboratory, with consistent regularity, generally about $1 \%-2 \%$ higher. The possible reasons are transportation and severe shocks; centrifugation and over time, red blood cells dissolve, resulting in a decrease in red blood cell volume. It suggests that when blood gas analysis indicates that the red blood cell volume has dropped to the standard for blood transfusion, clinicians should immediately take blood routine review and give the patient blood transfusion treatment in time. The correlation coefficient in this study is 0.93 , and in the above studies are all above 1, indicating that the red blood cell volume has a good correlation. The results of this study show the contradiction that the average hemoglobin value of blood gas analysis is higher than the central laboratory, while the average red blood cell volume is lower than the central laboratory. The hemoglobin measured by GEM premier 3000 is calculated by the formula (red blood cell volume * coefficient), which shows that the calculation coefficient of the GEM premier 3000 machine in this study is unreasonable and needs to be re-adjusted; on the other hand, whether the coefficient can be adjusted so that the t-test difference between GEM premier 3000 and the results of the central laboratory's hemoglobin test is not statistically significant, let the hemoglobin in the blood gas analysis result be the reference standard for applying for blood transfusion, thereby saving clinical manpower and material resources and patient costs.

\section{CONCLUSION}

GEM premier 3000 can quickly detect serum potassium, serum sodium, blood glucose, hemoglobin and red blood cell volume, which can provide a quick reference value for clinical judgment. According to the results of this study, the results of electrolytes are usually lower in blood gas analysis than in the laboratory; the results of blood glucose and red blood cell volume are usually higher in blood gas analysis than in the laboratory; the hemoglobin results are calculated according to the hematocrit* coefficient (adjustable) in the blood gas analysis. In this study, it was found that the GEM premier 3000 instrument coefficient setting was unreasonable, resulting in contradictory results; therefore, the reference value of the results of blood gas analysis for hemoglobin was lowered. According to the results of this study, the difference results between blood gas analysis and the central laboratory is statistically significant, so the judgment value of blood gas analysis cannot replace the clinical judgment of the central laboratory. Future research should be close to the actual clinical operation process, such as tracking the specific specimen collection and inspection time, and the impact of the intermediate time on the inspection results; pneumatic logistics is widely used in hospitals to transport specimens [14]; the violent shock process will affect the test results; with the diversification of rapid blood gas analyzer product types and test items, the standard reference values and meanings of various test results of blood gas analysis should be revised to provide a strong basis for clinicians to judge the results of rapid blood gas analysis. In the process of collecting and testing specimens, we should strengthen nurses' standardized operation training, test specimens as soon as possible, and at the same time, improve the effective test results of specimens by learning specimen quality detection events [15]. 


\section{CONFLICTS OF INTEREST}

The authors declare no conflicts of interest regarding the publication of this paper.

\section{REFERENCES}

1. Luo, K., Liang, S., Liu, Y., He, H., He, Y. and Huang, H. (2019) Correlation and Consistency of Electrolytes, Blood Glucose and Lactic Acid Test Results Detected by POCT Blood Gas Analyzer and Central Laboratory. International Journal of Laboratory Medicine, 40, 1938-1940+1944.

2. Zatloukal, J., Pouska, J., Kletecka, J., Pradl, R. and Benes, J. (2016) Comparison of the Accuracy of Hemoglobin Point of Care Testing Using Hemocue and GEM Premier 3000 with Automated Hematology Analyzer in Emergency Room. Journal of Clinical Monitoring and Computing, 30, 949-956. https://doi.org/10.1007/s10877-015-9799-z

3. Zhang, L., Zhu, Y., Ran, Y. and He, X. (2020) Discussion on the Consistency of Blood Gas Analyzer and Conventional Instruments in the Same Items. Chinese Journal of Laboratory Pathologist, 12, 77-80.

4. Chao, C.F. and Xu, Y. (2019) Research Progress on the Factors That Affect the Indwelling Time of Arterial Indwelling Needle and Nursing Countermeasures. Acta Academiae Medicinae Xuzhou, 39, 232-234.

5. Liu, X.M., Yang, M.L., Yi, J.Q. and Pan, X.X. (2015) Multifunctional Automatic Blood Cell Analyzer Sysmex XN-9000 Fluid Model Performance; Verification. China Medical Equipment, No. 4, 44-46.

6. Yu, M., Sun, Q. and Jiang, F. (2017) Performance Verification of VITROS 5600 Automatic Biochemical Analyzer. International Journal of Laboratory Medicine, 38, 56-60.

7. Westgard (2021) CLIA Requirements for Analytical Quality. Westgard. https://www.westgard.com/clia.htm

8. Xu, J.H., Liu, D.D., Huang, X.Z. and Zhuang, J.H. (2015) Application of New Guideline CLSI EP9-A3 in Methodological Comparison and Deviation Assessment. National Medical Journal of China, 95, 894-897.

9. Yu, J. (2016) Investigation on the Practical Application Significance of Non-Traditionary Test Items by GEM Premier 3000 Blood Gas Analyzer. Laboratory Medicine, No. 1, 26-30.

10. Xiong, W.-F., Xiao, G.-J., Liu, Y.-T., Yang, N., Cao, D. and Chen, S. (2018) Comparative Analysis of the Results of Non-Traditional Detection of GEM Premier 3000Blood Gas Analyzer and Conventional Instruments in Central Laboratory. Medical Information, 31, 160-162.

11. Zhong, G.Z. (2020) Regression Analysis of the Difference between GEM3000 Blood Gas Analyzer and Biochemical Analyzer in the Determination of Blood Potassium. China Practical Medical, 15, 197-198.

12. Liu, H., Ma, Y., Zhang, P., Zhang, Y. and Yang, B. (2012) Comparison of Determination of Blood Glucose by Blood Gas Analyzer, Portable Blood Glucose Meter and Automated Biochemical Analyzer. Laboratory Medicine and Clinic, 9, 407-410.

13. Spielmann, N., Mauch, J., Madjdpour, C., Schmugge, M., Weiss, M. and Haas, T. (2012) Accuracy and Precision of Hemoglobin Point-of-Care Testing during Major Pediatric Surgery. International Journal of Laboratory Hematology, 34, 86-90. https://doi.org/10.1111/j.1751-553x.2011.01363.x

14. Cui, F.Y. and Chen, H.W. (2017) Application Analysis of Medical Logistics System in Modern Hospital. China Medical Devices, 32, 137-140.

15. Li, T.M., Liang, S.X., Wang, S.X., et al. (2014) Research on Factors of Detection Accuracy of GEM Premier 3000 Blood Gas Analyzer. Hebei Medical Journal, No. 9, 1368-1370. 